\title{
Exploring the Crystal Structure Space of $\mathrm{CoFe}_{2} \mathrm{P}$ by Using Adaptive Genetic Algorithm Methods
}

\author{
P. Nieves ${ }^{1}$, S. Arapan ${ }^{1,2}$, and S. Cuesta-López ${ }^{1,3}$ \\ ${ }^{1}$ ICCRAM, International Research Center in Critical Raw Materials and Advanced Industrial Technologies, \\ University of Burgos, 09001 Burgos, Spain \\ ${ }^{2}$ Institute of Electronic Engineering and Nanoelectronics, Academy of Sciences of Moldova, MD-2028 Chişinău, Moldova \\ ${ }^{3}$ Advanced Materials, Nuclear Technology and Nanobiotechnology Department, University of Burgos, 09001 Burgos, Spain \\ Advances in theoretical and computational condensed matter physics have opened the possibility to predict and design magnetic \\ materials for specific technological applications. In this paper, we use the adaptive-genetic algorithm technique for exploring the \\ low-energy crystal structure configurations of $\mathrm{Co}_{0.25} \mathrm{Fe}_{0.5} \mathbf{P}_{\mathbf{0 . 2 5}}$, aiming to find new low-energy non-cubic phases with high saturation \\ magnetization that might be interesting for high-performance permanent magnet development.
}

Index Terms-Adaptive algorithms, genetic algorithms, magnetic materials, magnetic properties, permanent magnets.

\section{INTRODUCTION}

$\mathbf{E}$ XPERIMENTS have reported $\mathrm{Co}-\mathrm{Fe}-\mathrm{P}$ compounds as highly tuneable ferromagnetic alloys. For example, it has been shown that in this compound the magnetic anisotropy can be controlled by changing film thickness [1], Co concentration [2], and heat treatment [3]. Additionally, its saturation magnetization and coercivity can be modified by adjusting the bath composition and plating conditions in electroless deposition [4]. Moreover, the addition of $\mathrm{P}$ in $\mathrm{Fe}-\mathrm{Co}$ alloys also induces changes in their microstructure and electrical resistivity [5]. Recently, it has been found that $T_{\mathrm{C}}$ exhibits a strong compositional dependence in $\mathrm{Co}_{\mathrm{x}} \mathrm{Fe}_{2-\mathrm{x}} \mathrm{P}$ nanoparticles [6]. Although many of these phases are soft magnets, it is known that distorted Fe-Co alloys [7], [8] with very high anisotropy combined with the highest saturation magnetization of $2.4 \mathrm{~T}$ can lead to energy products with values exceeding $(\mathrm{BH})_{\max }=1.1 \mathrm{MJ} / \mathrm{m}^{3}$, which is nearly three times the value of the best $\mathrm{Nd}-\mathrm{Fe}-\mathrm{B}$ magnets. In particular, there is a special interest in the investigation of light metalloid substitution effects, such as $\mathrm{P}, \mathrm{N}$, or $\mathrm{C}$, in $\mathrm{Fe}-\mathrm{Co}$ systems, since they could promote the formation and stabilization of structures with large magneto-crystalline anisotropy. For example, recent experiments showed a giant magneto-crystalline anisotropy $\left(K=4.6 \mathrm{MJ} / \mathrm{m}^{3}\right)$ in $\mathrm{CoFe}_{2} \mathrm{C}$ [9]. Such compounds could be an alternative to current expensive rare-earth (RE) permanent magnets. In addition to possible industrial applications as a permanent magnet, $\mathrm{Co}-\mathrm{Fe}-\mathrm{P}$ nanocrystal-based alloys are presently used as metallic coating system on many manufactured products, replacing $\mathrm{Cr}$ due to its considerable health risk [10]. Further, Co-Fe-P alloys might be also suitable for future electro-magnetomechanical applications in micro-machine and nano-devices by functionalizing graphene aerogels [11].

Recently, the traditional way based on exploratory

Manuscript received March 9, 2017; accepted July 9, 2017. Date of publication July 18, 2017; date of current version October 24, 2017. Corresponding author: P. Nieves (e-mail: pnieves@ubu.es).

Color versions of one or more of the figures in this paper are available online at http://ieeexplore.ieee.org.

Digital Object Identifier 10.1109/TMAG.2017.2727880 syntheses of searching for new materials began to be replaced by computational approaches (promoted by efforts such as the materials genome initiative) [12]. At present time, taking advantage of accurate density functional theory (DFT) methods and high supercomputer performance, large open material databases, such as AFLOW [13], [14] and Materials Project [15], [16], have been created, providing a powerful tool for discovering and designing novel materials. One of the most important and hard task in this new strategy is the prediction of new stable or metastable phases. Many methods have been developed for predicting crystal structure as the simulated annealing [17], basin hopping [18], ab-initio random structure search [19], metadynamics [20], particle swarm optimization [21], cluster expansion method [22], and adaptive-genetic algorithms (AGA) [23]. Some of these methods have been implemented in available online codes like CALYPSO [24], based on the particle swarm optimization, XTALOPT [25] and USPEX [26], based on AGA. Recently, the cluster expansion method and AGA have been used to predict new RE-free magnetic crystal phases [27]-[30].

AGAs are adaptive heuristic algorithms which solve optimization problems based on evolutionary ideas of natural selection and genetics. AGA uses evolutionary techniques, such as inheritance, selection, mutation, and crossover to find structures very low in energy that might be stabilized experimentally. In this paper, we make use of an AGA technique for exploring low-energy crystal structure configurations of $\mathrm{Co}_{0.25} \mathrm{Fe}_{0.5} \mathrm{P}_{0.25}$. We have also calculated some basic properties by performing ab-initio calculations. Finally, we test the performance of AGA approach by comparing our results with the current available crystal structure database AFLOW [13], [14].

\section{Methodology}

The crystal structure prediction is computationally performed by using the USPEX code [26], which is an implementation of evolutionary-genetic algorithms. Initially, a set of structures (a population) is generated at random-the first generation. A new generation is created by applying various genetic mechanism to a subset of the most fit individuals. For

0018-9464 () 2017 IEEE. Translations and content mining are permitted for academic research only. Personal use is also permitted,

but republication/redistribution requires IEEE permission. See http://www.iee.org/publications_standards/publications/rights/index.html for more information. 
crystal structures at $T=0 \mathrm{~K}$ and $P=0 \mathrm{~Pa}$, the fitness criterion is the total energy of the system. Hence, to estimate the fitness, structures should undergo structural relaxation, which could be done by any numerical software performing structure optimization. Here, this task is done by using abinitio structure calculation software VASP [31]-[33]. USPEX is interfaced with VASP and provides a procedure to perform structures optimization. Relaxation is done stepwise, with a combination of ion positions and cell shape optimization, followed by a cell volume relaxation. One starts calculations with a low accuracy, since generated structures are highly distorted and far from their equilibrium. Calculations are repeated several times by increasing the accuracy of calculations at each step.

However, we have modified it in the case of exploring the structural space of magnetic materials. In this first stage, we do not consider the spin polarization of the structures, since the account for spin for highly distorted structures results in a slow electronic convergence or even the impossibility to obtain a converged electronic charge density. We have also found that initial relaxation is more efficient if we start from a larger volume and perform optimization with an increased energy cutoff. Spin polarization is switched on at the second stage, when a general relaxation (including the volume) is performed. At this stage, the cutoff energy is increased by $40 \%$ with respect to the default VASP value. At the third stage, we perform a set of volume calculations around the equilibrium volume. At each volume point, cell shape and ion positions are optimized with a cutoff energy increased by $20 \%$. The equilibrium volume and energy are estimated from fitting $E=E(V)$ to third-order Birch-Murnaghan EOS. In this way, we can explore various local minima and avoid being trapped in one of them. All VASP calculations have been done with the projector augmented wave (PAW) method with Perdew, Burke, and Ernzerfhof (PBE) 5.4 potentials [40], [41]. For the $k$-point mesh, we used an automatic generation scheme with scaling parameter increasing from 10 to 30 .

For the genetic evolution, we have used default parameters suggested by USPEX. Once the ground state energy of the system (fitness) is evaluated, USPEX applies a series of genetic operations to best $65 \%$ of structures creating the next generation. Fifty percent of all structures are generated by crossover and $30 \%$ by genetic mutations. Another $20 \%$ of structures are, again, generated at random to provide a certain structural diversity. Calculations continue until convergence criterion or the maximum number of generations is reached. A convergence criterion is, usually, a set of best structures persistent over several generations. Due to the stochastic nature of the evolution process, several simulations should be run to get consistent results. Since we do not know a priori the number of atoms in the unit cell of stable phases, the entire process is repeated by considering structures containing an integer number of formula units. In our case of $\mathrm{Co}_{0.25} \mathrm{Fe}_{0.5} \mathrm{P}_{0.25}$, we have started exploring unit cells with four atoms $\left(\mathrm{CoFe}_{2} \mathrm{P}\right)$; then, we extended the study to eight atoms/unit cell $\left(\mathrm{Co}_{2} \mathrm{Fe}_{4} \mathrm{P}_{2}\right)$. However, increasing the number of atoms becomes computationally very demanding. Doubling the number of atoms doubles the number of structures in a generation, from 10
TABLE I

Properties of Low-Energy $\mathrm{Co}_{0.25} \mathrm{Fe}_{0.5} \mathrm{P}_{0.25}$ Structures Found in AFLOW Database. Their Crystal LatTice Structures Are ShOWEd IN Fig. 1

\begin{tabular}{cccccccc}
\hline Struct. Ref. Space & $\begin{array}{c}\mathrm{E}_{\text {cell }} / \text { atom } \\
\text { Group } \\
(\mathrm{eV} / \text { atom })\end{array}$ & $\begin{array}{c}\mathrm{V}_{\text {cell }} / \text { atom } \\
\left(\mathrm{A}^{3} / \text { ion }\right)\end{array}$ & $\begin{array}{c}\mu_{0} \mathrm{M}_{\mathrm{S}} \\
(\mathrm{T})\end{array}$ & $\begin{array}{c}\mathrm{B} \\
(\mathrm{GPa})\end{array}$ & $\mathrm{B}^{\prime}$ \\
\hline$\# 1$ & {$[34]$} & 62 & -7.53176 & 11.188 & 1.07 & 189.6 & 4.0 \\
$\# 2$ & {$[35]$} & 216 & -7.43907 & 10.850 & 1.50 & 197.8 & 6.0 \\
$\# 3$ & {$[36]$} & 139 & -7.41389 & 10.831 & 1.31 & 188.1 & 6.2 \\
$\# 4$ & {$[37]$} & 123 & -7.39385 & 10.869 & 1.22 & 194.5 & 6.1 \\
$\# 5$ & {$[38]$} & 99 & -7.38626 & 11.004 & 1.33 & 192.7 & 5.6 \\
\hline
\end{tabular}
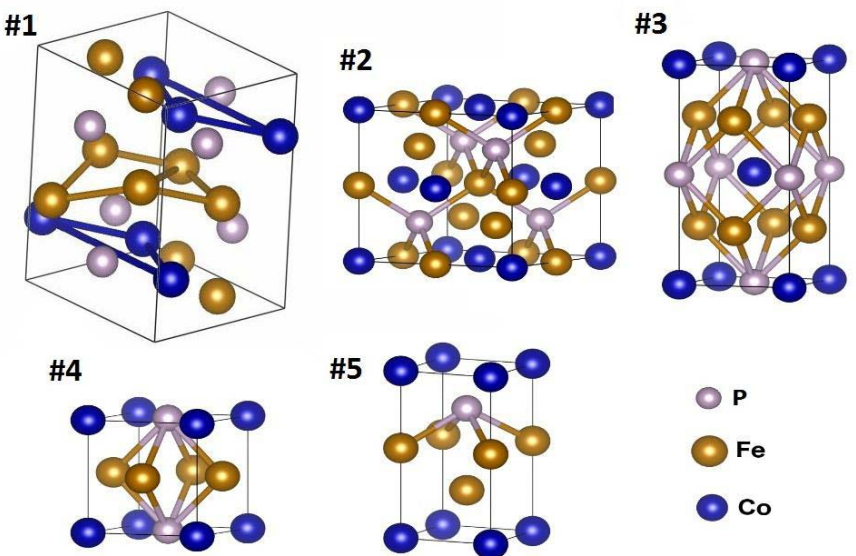

\#5

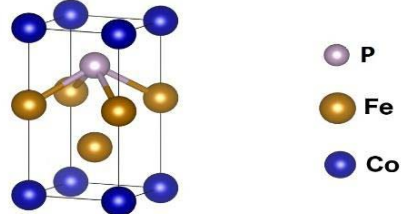

Fig. 1. Crystal lattices of low-energy $\mathrm{Co}_{0.25} \mathrm{Fe}_{0 .} \mathrm{P}_{0.25}$ structures found in AFLOW database. Some properties of them are given by Table I. Structure \#1 actually has four formula units.

for one formula unit to 20 for two formula units. But the most time and resources consuming part comes from ab-initio calculations, which scales as a cube of number of atoms.

\section{RESULTS}

We started by performing a search through the AFLOW database for known $\mathrm{Co}_{0.25} \mathrm{Fe}_{0.5} \mathrm{P}_{0.25}$ structures. We have found a set of calculated structures with negative enthalpy of formation. For the sake of comparing properties of these structures with those obtained by AGA exploration, we have recalculated structural parameters and basic physical properties with the same PAW PBE 5.4 potentials, cutoff energy, and $k$-points mesh. Properties at equilibrium conditions are obtained by performing standard energy calculations for a set of volumes, and fitting $E=E(V)$ results to the thirdorder Birch-Murnaghan equation of state (EOS). In Table I, we show obtained parameters of the EOS for the five lowest energy AFLOW structures, as well as the information about their space group, and saturation magnetization $\mu_{0} M_{\mathrm{S}}$. For the sake of clarity, we provide a graphical representation of unit cells of corresponding structures in Fig. 1.

After getting a set of reference structures, we proceeded to AGA exploration of the structural space of the $\mathrm{Co}_{0.25} \mathrm{Fe}_{0.5} \mathrm{P}_{0.25}$ systems. We have performed three simulations: two with one 


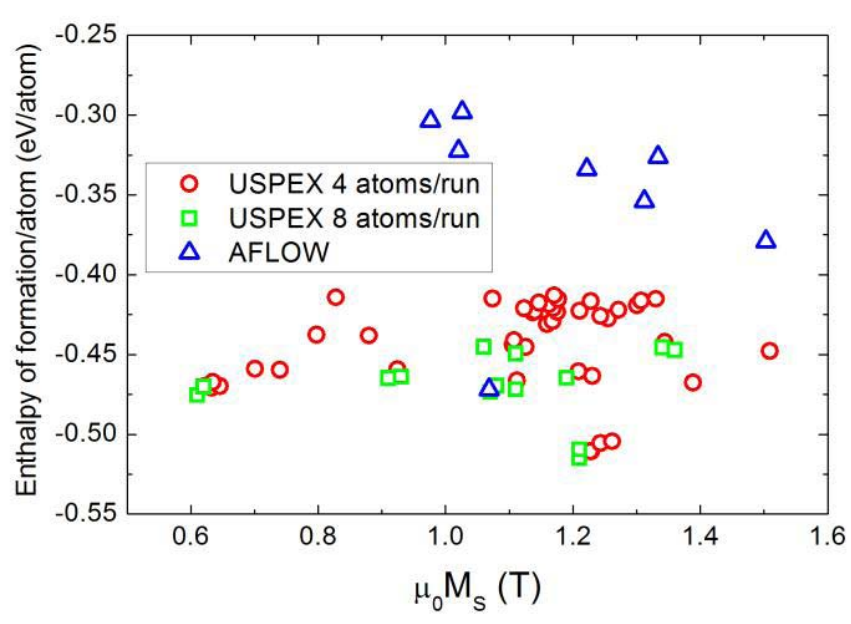

Fig. 2. Enthalpy of formation versus saturation magnetization for $\mathrm{Co}_{0.25} \mathrm{Fe}_{0.5} \mathrm{P}_{0.25}$ structures found in the AFLOW (blue triangles), and generated by USPEX during four (red circles) and eight (green squares) atoms/run.

formula unit $\left(\mathrm{CoFe}_{2} \mathrm{P}\right.$ and four atoms/cell) and one with two formula units $\left(\mathrm{Co}_{2} \mathrm{Fe}_{4} \mathrm{P}_{2}\right.$, eight atoms/cell). We have selected structures with negative enthalpy of formation and for all relevant structures, both from the AFLOW database and AGA search we show their enthalpy of formation versus saturation magnetization in Fig. 2. This figure provides in an intuitive way the main message of our research. The exploration of structural space by using a combined AGA and DFT approach provides us with a plethora of new phases with better properties than known structures. We can easily see that, already, in our first attempt with one formula unit (data shown in red circles), we obtained a set of structures that have much lower energy than reference structures (data shown in blue triangles).

For the second run with one formula unit, we used in the first generation a set of best structures obtained from the first run, which resulted in getting new structures with lower energies. We did not use high convergence criterion for the first run, and the result of the second run shows that our search is not converged. We have decided to enlarge the exploration space by increasing the number of atoms in the unit cell. The third run yielded another set of low-energy phases (data shown in green squares). We can see a small cluster comprising five best predicted structures, separated from the rest, but being very close in energy. These structures are graphically shown in Fig. 3 and their corresponding properties are given in Table II. In addition, we show the change in enthalpy about the equilibrium with respect to the energetically best structure in Fig. 4. This energy proximity is, probably, an indication that we need to continue our search for the stable structure of $\mathrm{Co}_{0.25} \mathrm{Fe}_{0.5} \mathrm{P}_{0.25}$. It is also possible, that the ground state structure of $\mathrm{Co}_{0.25} \mathrm{Fe}_{0.5} \mathrm{P}_{0.25}$ could be a disordered alloy, so by increasing the number of atoms in the unit cell we will get some approximants of the stable phase. However, increasing the number of atoms makes the current search computationally very demanding. One way to reduce the computational cost at the expense of some accuracy could be a combined DFT and classical force-fields approach [39].

One of the requirements for a structure to be suitable for permanent magnet applications is its geometry: usually
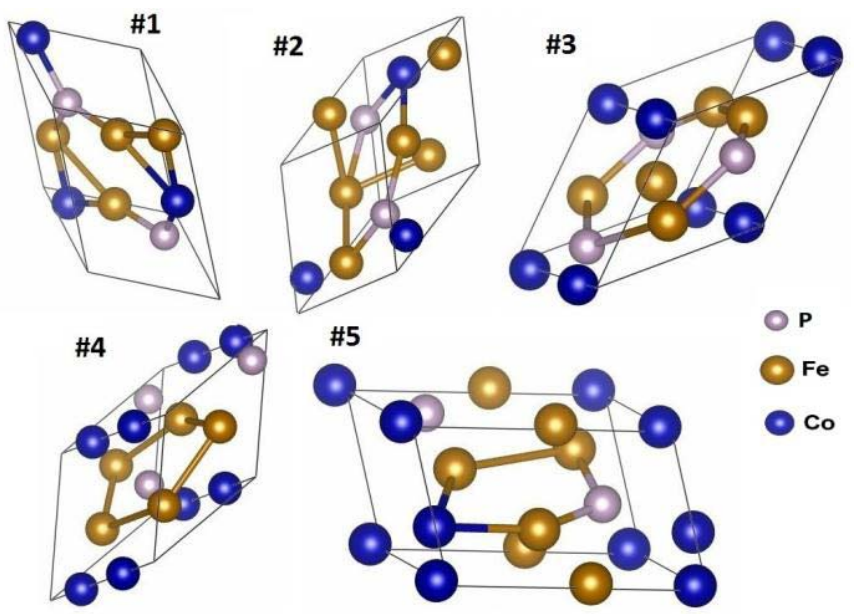

Fig. 3. Crystal lattices of low-energy $\mathrm{Co}_{0.25} \mathrm{Fe}_{0.5} \mathrm{P}_{0.25}$ structures obtained by USPEX calculations. Some properties of them are given by Table II.

TABLE II

Properties of Low-Energy $\mathrm{Co}_{0.25} \mathrm{Fe}_{0.5} \mathrm{P}_{0.25}$ Structures CALCUlated By USPEX DURING THE FOUR AND EIGHT Cell/Atoms Run. Their Crystal Lattice Structures Are Showed IN FIG. 3

\begin{tabular}{cccccccc}
\hline Struct & $\begin{array}{c}\text { USPEX } \\
\text { atoms/run }\end{array}$ & $\begin{array}{c}\text { Space } \\
\text { Group }\end{array}$ & $E_{\text {cell }} /$ atom & $\mathrm{V}_{\text {cell }} /$ atom & $\mu_{0} \mathrm{M}_{\mathrm{S}}$ & $\mathrm{B}$ & $\mathrm{B}^{\prime}$ \\
$\left(\mathrm{A}^{3} /\right.$ ion $)$ & $(\mathrm{T})$ & & \\
\hline$\# 1$ & 8 & 36 & -7.5748 & 11.205 & 1.21 & 191.9 & 5.7 \\
$\# 2$ & 4 & 36 & -7.57075 & 11.219 & 1.23 & 191.1 & 5.5 \\
$\# 3$ & 8 & 38 & -7.5695 & 11.210 & 1.21 & 187.2 & 6.2 \\
$\# 4$ & 4 & 38 & -7.56565 & 11.233 & 1.24 & 188.6 & 4.9 \\
$\# 5$ & 4 & 8 & -7.5644 & 11.256 & 1.26 & 173.7 & 3.8 \\
\hline
\end{tabular}

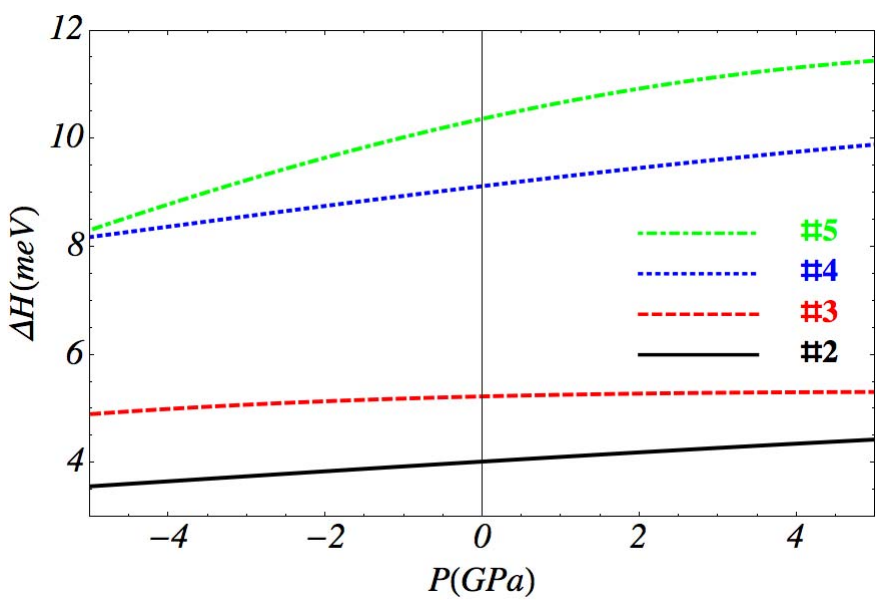

Fig. 4. Enthalpy difference $(\Delta H)$ versus pressure with respect to the most stable phase of $\mathrm{Co}_{0.25} \mathrm{Fe}_{0.5} \mathrm{P}_{0.25}$ (structure \#1) generated by USPEX (see Table II).

uniaxial structures within tetragonal or hexagonal symmetry show good magnetic anisotropy. We have redrawn Fig. 2 to show how the relation between the enthalpy of formation and 


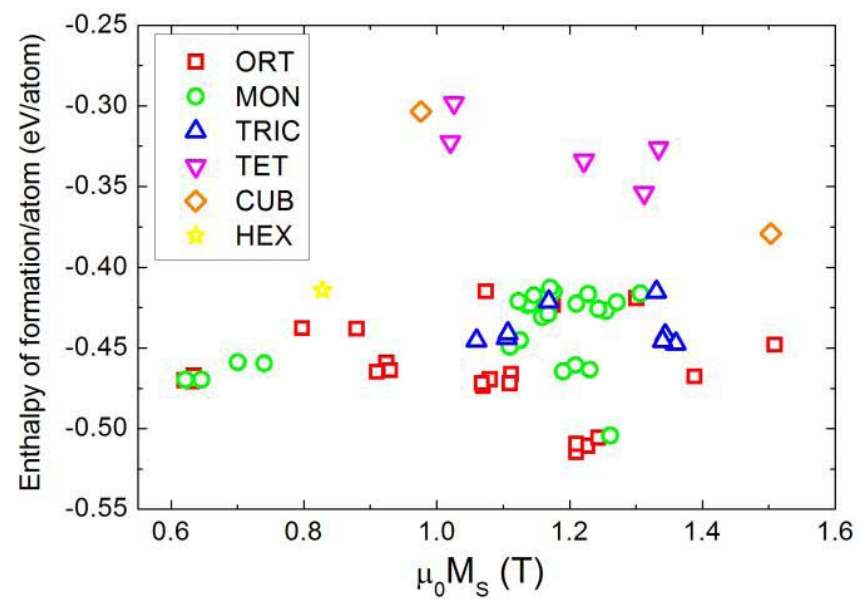

Fig. 5. Enthalpy of formation versus saturation magnetization for $\mathrm{Co}_{0.25} \mathrm{Fe}_{0.5} \mathrm{P}_{0.25}$ structures found in the AFLOW and generated by USPEX where the lattice system of each structure is shown with different symbols.

saturation magnetization correlates with the symmetry of the lattice. Results are shown in Fig. 5, where symbols showing data are associated with a certain symmetry class. We observe that most of the low-energy structures have an orthorhombic or monoclinic symmetry. Thus, at this stage it is difficult to draw a conclusion if $\mathrm{Co}_{0.25} \mathrm{Fe}_{0.5} \mathrm{P}_{0.25}$ could form in a structure yielding good magnetic anisotropy. A further exploration of the structural space is necessary with a subsequent calculation of magnetic anisotropy.

\section{CONCLUSION}

In summary, we have performed a search for new magnetic $\mathrm{Co}_{0.25} \mathrm{Fe}_{0.5} \mathrm{P}_{0.25}$ phases by applying structure predicting methods based on AGA. Our preliminary results show that these methods reveal a large set of previously unknown structures with energies lower than those provided by existing databases. Some of the predicted new phases exhibit noncubic structure with high saturation magnetization $(>1 \mathrm{~T})$, which could be an indication that we may find structures suitable for permanent magnet applications within $\mathrm{Co}-\mathrm{Fe}-\mathrm{P}$ systems. From these preliminary tests, we can conclude that genetic algorithms provide an efficient way to explore unknown magnetic phases, showing that they can reproduce and extend available databases, like AFLOW.

\section{ACKNOWLEDGMENT}

This work was supported in part by the NOVAMAG project under Grant 686056, in part by the EU Horizon 2020 Framework Program for Research and Innovation (2014-2020), and in part by the Spanish Supercomputing Network and CESVIMA for providing computational resources under Grant QCM-2016-2-0034.

\section{REFERENCES}

[1] E. A. Vasilyev, T. M. Tkachenko, V. M. Fedosyuk, O. I. Kasyutich, and A. P. Saiko, "Investigation of perpendicular magnetic anisotropy in Co-Fe-P/Cu multilayers by Mössbauer effect," J. Magn. Magn. Mater, vol. 111, pp. 34-38, Dec. 1992.
[2] S. Kumar, A. Krishnamurthy, and B. K. Srivastava, "On nature of magnetism in ferromagnetic alloys $\left(\mathrm{Fe}_{1-x} \mathrm{Co}_{x}\right)_{2} \mathrm{P}$," J. Phys. D, Appl. Phys., vol. 41, no. 5, p. 055001, Feb. 2008.

[3] J. Wei, E. Feng, L. Hao, D. Cao, J. Wang, and Q. Liu, "The influence of magnetic heat treatment on morphology, structure, magnetic properties of Fe-Co-P alloy films," Appl. Phys. A, vol. 115, pp. 359-363, Apr. 2014.

[4] W. Q. Huang, G. F. Huang, B. Liang, and C. L. Xie, "Magnetic properties of $\mathrm{CoFeP}$ films prepared by electroless deposition," J. Magn. Magn. Mater, vol. 321, pp. 1177-1181, May 2009.

[5] H. Taslimi, M. S. Heydarzadeh, S. Mehrizi, and M. Saremi, "Studies of the effects of addition of $\mathrm{P}$ and $\mathrm{Cr}$ on microstructure and electrical resistivity of nanocrystalline CoFe thin films," J. Mater. Sci., Mater. Electron., vol. 26, pp. 2962-2968, May 2015.

[6] D. Li, M. P. Arachchige, B. Kulikowski, G. Lawes, T. Seda, and S. L. Brock, "Control of composition and size in discrete $\mathrm{Co}_{x} \mathrm{Fe}_{2-x} \mathrm{P}$ nanoparticles: Consequences for magnetic properties," Chem. Mater. vol. 28, no. 11, pp. 3920-3927, May 2016.

[7] T. Burkert, L. Nordstrom, O. Eriksson, and O. Heinonen, "Giant magnetic anisotropy in tetragonal FeCo alloys," Phys. Rev. Lett., vol. 93, p. 027203, Jul. 2004.

[8] G. Andersson et al., "Perpendicular magnetocrystalline anisotropy in tetragonally distorted Fe-Co alloys," Phys. Rev. Lett., vol. 96, p. 037205 , Jan. 2006.

[9] A. A. El-Gendy, M. Bertino, D. Clifford, M. Qian, N. S. Khanna, and E. Everett Carpenter, "Experimental evidence for the formation of $\mathrm{CoFe}_{2} \mathrm{C}$ phase with colossal magnetocrystalline-anisotropy," Appl. Phys. Lett., vol. 106, p. 213109, May 2015.

[10] R. W. Revie and U. Erb, Corrosion Behavior of Electrodeposited Nanocrystals. 3rd ed. Hoboken, NJ, USA: Wiley, 2011.

[11] G.-P. Zheng, X. Lu, and Z. Han, "Synthesis and electro-magnetomechanical properties of graphene aerogels functionalized with Co-Fe-P amorphous alloys," Micromachines, vol. 7, p. 117, Jul. 2016

[12] Z. Liu, "Perspective on Materials Genome," Chin. Sci. Bull., vol. 59, no. 15, pp. 1619-1623, Jan. 2014.

[13] Automatic-FLOW for Materails Discovery (AFLOW). Accessed on Feb. 25, 2017. [Online]. Available: http://aflowlib.org/

[14] S. Curtarolo et al., "AFLOWLIB.ORG: A distributed materials properties repository from high-throughput $\mathrm{AB}$ initio calculations," Comput. Mater. Sci., vol. 58, p. 227, Jun. 2012.

[15] A. Jain et al., "The materials project: A materials genome approach to accelerating materials innovation," APL Mater., vol. 1, p. 011002, Jul. 2013 .

[16] The Materials Project. Accessed on Feb. 25, 2017. [Online]. Available: https://materialsproject.org/

[17] S. Kirkpatrick, C. D. Gelatt, and M. P. Vecchi, "Optimization by Simulated Annealing," Science, vol. 220, no. 4598, p. 671, May 1983.

[18] D. J. Wales and J. P. K. Doye, "Global optimization by basin-hopping and the lowest energy structures of Lennard-Jones clusters containing up to 110 atoms," J. Phys. Chem. A, vol. 101, p. 5111, Jul. 1997.

[19] C. J. Pickard and R. J. Needs, "Abinitio random structure searching," J. Phys., Condens. Matter, vol. 23, no. 5, p. 053201, 2011.

[20] P. Raiteri, R. Martoňák, and M. Parrinello, "Exploring polymorphism: The case of benzene," Angew. Chem. Int. Edit., vol. 44, no. 24, pp. 3769-3773, Jun. 2005.

[21] Y. Wang, J. Lv, L. Zhu, and Y. Ma, "Crystal structure prediction via particle-swarm optimization," Phys. Rev. B, Condens. Matter, vol. 82, p. 094116, Sep. 2010.

[22] A. Seko, Y. Koyama, and I. Tanaka, "Cluster expansion method for multicomponent systems based on optimal selection of structures for density-functional theory calculations," Phys. Rev. B, Condens. Matter, vol. 80, p. 165122 , Oct. 2009.

[23] M. Ji, K. Umemoto, C. Z. Wang, K. M. Ho, and R. M. Wentzcovitch, "Ultrahigh-pressure phases of $\mathrm{H}_{2} \mathrm{O}$ ice predicted using an adaptive genetic algorithm," Phys. Rev. B, Condens. Matter, vol. 84, p. 220105 , Dec. 2011.

[24] Y. Wang, J. Lv, L. Zhu, and Y. Ma, "CALYPSO: A method for crystal structure prediction," Comput. Phys. Commun., vol. 183, p. 2063 , Oct. 2012.

[25] D. C. Lonie and E. Zurek, "XtalOpt: An open-source evolutionary algorithm for crystal structure prediction," Comput. Phys. Commun., vol. 182, pp. 372-387, Feb. 2011.

[26] A. O. Lyakhov, A. R. Oganov, H. T. Stokes, and Q. Zhu, "New developments in evolutionary structure prediction algorithm USPEX," Comput. Phys. Commun., vol. 184, p. 1172, Apr. 2013.

[27] A. Díz-Ortiz, R. Drautz, M. Fahnle, H. Dosch, and J. M. Sanchez, "Structure and magnetism in bcc-based iron-cobalt alloys," Phys. Rev. B, Condens. Matter, vol. 73, p. 224208, Jun. 2006. 
[28] R. Drautz, A. Díaz-Ortiz, M. Fähnle, and H. Dosch, "Ordering and magnetism in Fe-Co: Dense sequence of ground-state structures," Phys. Rev. Lett., vol. 93, p. 067202, Aug. 2004.

[29] M. C. Nguyen, X. Zhao, M. Ji, C.-Z. Wang, B. Harmon, and K.-M. Ho, "Atomic structure and magnetic properties of $\mathrm{Fe}_{1-x} \mathrm{Co}_{x}$ alloys," J. Appl. Phys., vol. 111, p. 07E338, Mar. 2012.

[30] X. Zhao et al., "Exploring the structural complexity of intermetallic compounds by an adaptive genetic algorithm," Phys. Rev. Lett., vol. 112 p. 045502, Jan. 2014.

[31] G. Kresse and J. Hafner, "Abinitio molecular dynamics for liquid metals," Phys. Rev. B, Condens. Matter, vol. 47, p. 558, Jan. 1994.

[32] G. Kresse and J. Furthmüller, "Efficiency of ab-initio total energy calculations for metals and semiconductors using a plane-wave basis set," Comput. Mater. Sci., vol. 6, pp. 15-50, Jul. 1996.

[33] G. Kresse and J. Furthmüller, "Efficient iterative schemes for ab initio total-energy calculations using a plane-wave basis set," Phys. Rev. B, Condens. Matter, vol. 54, p. 11169, Oct. 1996.

[34] Automatic-FLOW for Materails Discovery (AFLOW). Accessed on Feb. 25, 2017. [Online]. Available: http://aflowlib.duke.edu/ AFLOWDATA/LIB3_RAW/CoFe_pvP/ICSD_603579.ABC/
[35] Automatic-FLOW for Materails Discovery (AFLOW). Accessed on Feb. 25, 2017. [Online]. Available: http://aflowlib.duke.edu/ AFLOWDATA/LIB3_RAW/CoFe_pvP/T0002.AB2C/

[36] Automatic-FLOW for Materails Discovery (AFLOW). Accessed on Feb. 25, 2017. [Online]. Available: http://aflowlib.duke.edu/ AFLOWDATA/LIB3 RAW/CoFe pvP/TFCC013.BCA/

[37] Automatic-FLOW for Materails Discovery (AFLOW). Accessed on Feb. 25, 2017. [Online]. Available: http://aflowlib.duke.edu/ AFLOWDATA/LIB3_RAW/CoFe_pvP/TFCC016.BCA/

[38] Automatic-FLOW for Materails Discovery (AFLOW). Accessed on Feb. 25, 2017. [Online]. Available: http://aflowlib.duke.edu/ AFLOWDATA/LIB3_RAW/CoFe_pvP/TBCC006.BCA/

[39] S. Q. Wu et al., "An adaptive genetic algorithm for crystal structure prediction," J. Phys., Condens. Matter, vol. 26, no. 3, p. 035402 , Dec. 2013.

[40] G. Kresse and D. Joubert, "From ultrasoft pseudopotentials to the projector augmented-wave method," Phys. Rev. B, Condens. Matter, vol. 59, p. 1758, Jan. 1999.

[41] J. P. Perdew, K. Burke, and M. Ernzerhof, "Generalized Gradient Approximation Made Simple," Phys. Rev. Lett., vol. 77, p. 3865, Oct. 1996 